\title{
Prostate cancer with disseminated carcinomatosis of the bone marrow: Two case reports
}

\author{
TASUKU HIROSHIGE and YOSHIRO EGUCHI \\ Department of Urology, Kurume St. Mary's Hospital, Kurume, Fukuoka 830-0042, Japan
}

Received March 9, 2017; Accepted May 10, 2017

DOI: $10.3892 / \mathrm{mco} .2017 .1303$

\begin{abstract}
Disseminated carcinomatosis of the bone marrow (DCBM) is caused by cancer metastasis to the bone marrow and is often accompanied by disseminated intravascular coagulation (DIC), with rapid clinical progression. We herein report two cases receiving treatment with combined androgen blockade $(\mathrm{CAB})$ and denosumab for prostate cancer with DCBM. The patient in case 1 was an 80-year-old man who was admitted with a 3-day history of melena and was diagnosed with prostate cancer with DCBM by bone marrow biopsy. Despite receiving therapy with $\mathrm{CAB}$ and denosumab, the patient developed castration-resistant prostate cancer (CRPC) with accompanying relapse of DIC and succumbed to the disease 7 months later. The patient in case 2 was a 64-year-old man who was admitted with bleeding after a tooth extraction. After a diagnosis of prostate cancer with DCBM, CAB therapy was administered. Enzalutamide was administered following development of CRPC, and the patient has not since experienced an increase in prostate-specific antigen, recurrence of anemia or DIC. To the best of our knowledge, these are the first documented cases in which DCBM was treated with denosumab and enzalutamide.
\end{abstract}

\section{Introduction}

Disseminated carcinomatosis of the bone marrow (DCBM) is caused by cancer metastasis to the bone marrow and is often accompanied by disseminated intravascular coagulation (DIC), leukoerythroblastosis and microangiopathic hemolytic anemia. The clinical course is rapid. The majority of DCBM cases originate from the stomach (1), whereas carcinomatosis arising from the prostate is rare. Several reports indicate that combined androgen blockade (CAB) therapy was used as the

Correspondence to: Dr Tasuku Hiroshige, Department of Urology, Kurume St. Mary's Hospital, 422 Tsubukuhonmachi, Kurume, Fukuoka 830-8543, Japan

E-mail: tkkn19841220@yahoo.co.jp

Key words: prostate cancer, disseminated carcinomatosis of the bone marrow, disseminated intravascular coagulation, denosumab, enzalutamide primary therapy for prostate cancer with DCBM, similar to common metastatic prostate cancer, and zoledronic acid was often used in combination with $\mathrm{CAB}$ for bone metastasis. However, to the best of our knowledge, the use of denosumab, another agent used for bone metastasis, for the treatment of disseminated carcinomatosis has not been previously reported. In cases of prostate cancer with DCBM, docetaxel treatment may be difficult due to myelosuppression or DIC following progression to castration-resistant prostate cancer (CRPC). It is possible to administer novel hormonal agents, such as enzalutamide, in such cases. We herein report two cases of prostate cancer with DCBM treated with a combination of $\mathrm{CAB}$ and denosumab.

\section{Case reports}

Case 1. An 80-year-old man presented to a local physician with a 3-day history of melena and he was rushed to Kurume St. Mary's Hospital (Kurume, Japan) with suspected gastrointestinal bleeding in May 2016. Physical examination revealed pallor of the palpebral conjunctiva, indicating anemia. Laboratory tests revealed a hemoglobin level of $4.4 \mathrm{~g} / \mathrm{dl}$, a hematocrit of $13.7 \%$, a platelet count of $97 \times 10^{3} / \mu 1$, an unmeasurable prothrombin time (PT) of $>140 \mathrm{sec}$, a fibrinogen level of $410.4 \mathrm{mg} / \mathrm{dl}$, a fibrin degradation products (FDP) level of $14.7 \mu \mathrm{g} / \mathrm{ml}$, a lactate dehydrogenase (LDH) level of 2,662 IU/1, and an alkaline phosphatase (ALP) level of 4,389IU/1. Esophagogastroduodenoscopy and colonoscopy were performed, but did not reveal a significant bleeding focus. The patient was transfused with 4 units of packed red blood cells (PRBCs) for anemia at the first medical examination. However, the hemoglobin level only increased to $5.7 \mathrm{~g} / \mathrm{dl}$. Pancytopenia and DIC developed. Furthermore, immature cells were identified in the peripheral blood. A bone marrow biopsy was performed, as a hematological disorder was suspected based on these findings. The results of the histopathological examination revealed bone marrow metastasis of adenocarcinoma, and immunostaining for prostate-specific antigen (PSA) was positive (Fig. 1A and B). Subsequently, an enhanced whole-body computed tomography (CT) scan revealed an edematous prostate with a heterogeneous internal structure; multiple lymphadenopathy was identified in the mediastinum, the hilum and the inferior lobe of the right lung, and the iliac region. Moreover, bone scintigraphy revealed multiple bone metastases (Fig. 2A and B). Based on the 
imaging findings, the PSA level was measured and found to be $39.77 \mathrm{ng} / \mathrm{ml}$. Prostate cancer (cTxN1M1b) with DCBM was diagnosed from these findings. Combination treatment with CAB (degarelix at an initial dose of $240 \mathrm{mg}$ and a maintenance dose of $160 \mathrm{mg}$ monthly, and bicalutamide $80 \mathrm{mg} /$ day) and denosumab (120 mg/day monthly) was initiated. Over the next 7 days, the patient received 4 units of PRBCs. Two weeks after treatment initiation, anemia and DIC improved. A needle biopsy of the prostate gland was performed 5 months after the initial diagnosis and showed Gleason 5+4=9 adenocarcinoma. After 1 month of combination treatment with $\mathrm{CAB}$ and denosumab, the PSA level decreased to $27.11 \mathrm{ng} / \mathrm{ml}$. However, the PSA level gradually increased to $141.50 \mathrm{ng} / \mathrm{ml} 6$ months after treatment initiation. In addition, pancytopenia and DIC recurred. The patient ultimately succumbed to cancer-related multi-organ failure in December 2016.

Case 2. A 64-year-old man presented to a local doctor in December 2014 with impaired hemostasis following a tooth extraction. The patient was referred to Kurume St. Mary's Hospital as his blood tests revealed pancytopenia and DIC. The findings on physical examination were unremarkable. The laboratory tests revealed a hemoglobin level of $7.4 \mathrm{~g} / \mathrm{dl}$, a hematocrit of $22 \%$, a platelet count of $75 \times 10^{3} / \mu 1$, a PT of $80.4 \mathrm{sec}$, a fibrinogen level of $170.1 \mathrm{mg} / \mathrm{dl}$, an FDP level of $23.0 \mu \mathrm{g} / \mathrm{ml}$, an LDH level of $818 \mathrm{IU} / 1$ and an ALP level of $511 \mathrm{IU} / 1$. A bone marrow biopsy was performed, and the pathological examination revealed bone marrow metastasis from adenocarcinoma; immunostaining for PSA was positive (Fig. 1C and D). The patient's PSA level was 11,951 ng/ml. Pelvic magnetic resonance imaging and enhanced whole-body CT scan revealed prostate cancer with right seminal vesicle invasion and metastasis to the internal iliac lymph nodes. Bone scintigraphy revealed diffuse bone metastases (Fig. 2 and D). DCBM originating from prostate cancer (cT3bN1M1b) was diagnosed based on these findings. Combination treatment with CAB (degarelix, initial dose of $240 \mathrm{mg}$ and maintenance dose of $160 \mathrm{mg}$ monthly; and bicalutamide, $80 \mathrm{mg} /$ day) and denosumab (120 mg monthly) was initiated. On the day following the first admission, the patient received 2 units of PRBCs. Anemia and DIC improved after treatment initiation. Two months after the diagnosis, needle biopsy of the prostate was performed, showing Gleason $4+5=9$ adenocarcinoma. The PSA decreased to $15.47 \mathrm{ng} / \mathrm{ml}$ after 4 months of combination treatment with $\mathrm{CAB}$ and denosumab. Two months later, however, the PSA increased to $70.57 \mathrm{ng} / \mathrm{ml}$ and flutamide (375 mg/day) was administered instead of bicalutamide. Although the nadir PSA level reached $6.85 \mathrm{ng} / \mathrm{ml}$, it increased to $15.44 \mathrm{ng} / \mathrm{ml} 2$ months after the administration of flutamide. Therefore, the patient was started on enzalutamide (160 mg/day), and he has not since exhibited an increase in PSA, recurrence of anemia or DIC.

\section{Discussion}

Jacho et al reported 'diffuse infiltrative carcinoma' as a type of bone metastasis in 1930 (2). Based on that report, Hayashi et al advocated DCBM from the clinicopathological characteristics using the count result of diffuse bone marrow metastasis from solid cancers in Japan in 1979 (1). Gastric cancer accounts for
$>90 \%$ of all primary lesions, while prostate cancer comprises only $3.7 \%$ (1). The common clinical manifestations of DCBM are backache, anemia and bleeding tendency. In addition, DCBM is frequently complicated by the appearance of immature polymorphs or erythroblasts in the peripheral blood, DIC or microangiopathic hemolytic anemia in the laboratory findings, and an increase in LDH and ALP levels in the majority of the cases. With respect to tumor growth in the form of bone marrow metastasis on pathological examination, diffuse infiltration is dominant, rather than tuberculated growth. Although a definitive diagnosis of this disease requires histological confirmation, such as bone marrow aspiration or biopsy, bone marrow aspiration for DCBM was a dry tap in 50\% (1) and cancer cells were observed in only $\sim 60 \%$ of the cases of DCBM for which bone marrow aspiration or biopsy was performed (3). Bone metastasis frequently develops in prostate cancer, and it is reported that $>25 \%$ of advanced cancer cases are complicated by DIC (4). The majority of prostate cancer cases with accompanying DIC become terminal. Therefore, a diagnosis requiring highly invasive examinations is often not considered. Thus, DCBM may occur more frequently than what has been reported. Chernow and Wallner recommend performing a bone scintigram and bone marrow biopsy, due to the high possibility of bone marrow metastasis if there are more than two findings among leukoerythroblastosis, platelet count $<100,000 / \mu 1, \mathrm{LDH}>500 \mathrm{IU} / 1$ and/or bone pain (5).

As regards bone metastasis, fibrosis, necrosis, osteoid formation and a change of the hematopoietic function in the peripheral bone marrow are observed. The bone reactions in the metastatic lesions are classified as osteoblastic, osteolytic, intertrabecular, or mixed-type metastasis. Intertrabecular metastasis is a concept similar to DCBM. In the case of intertrabecular metastasis, cancer cells are present between the bone trabeculae, and the bone cortex is preserved without destruction. The cancer cells replace the bone cells and spread. Intertrabecular metastasis is the initial presentation of bone metastasis, and may later transform to other patterns (6). However, intertrabecular metastasis leads to DIC when it progresses without change. Older age, male gender, primary tumor necrosis and advanced stage were found to be risk factors for DIC in solid tumors (7).

The degree of fibrinolytic activation differs among DIC types; DIC is classified as one of three types: With suppressed fibrinolysis, with enhanced fibrinolysis, and an intermediate type between the other two. The three major diseases associated with the development of DIC are sepsis, acute leukemia and solid cancers. The type of DIC associated with DCBM appears to be DIC with enhanced fibrinolysis, which develops due to tissue factors in cancer cells activating exogenous coagulation. In cases of sepsis, DIC develops due to the presence of inflammatory cytokines, such as lipopolysaccharide, tumor necrosis factor and interleukin-1 in the blood vessels, and secretion of plasminogen activator inhibitor by monocytes or vascular endothelial cells, thereby activating coagulation. Expression of thrombomodulin, which exerts anticoagulant effects, is suppressed in the vascular endothelial cell in this exigency. Thus, coagulation is aggravated and thrombi are formed in the microvasculature. Therefore, organ dysfunction may be the main presenting symptom. By contrast, in cases of DIC 
A
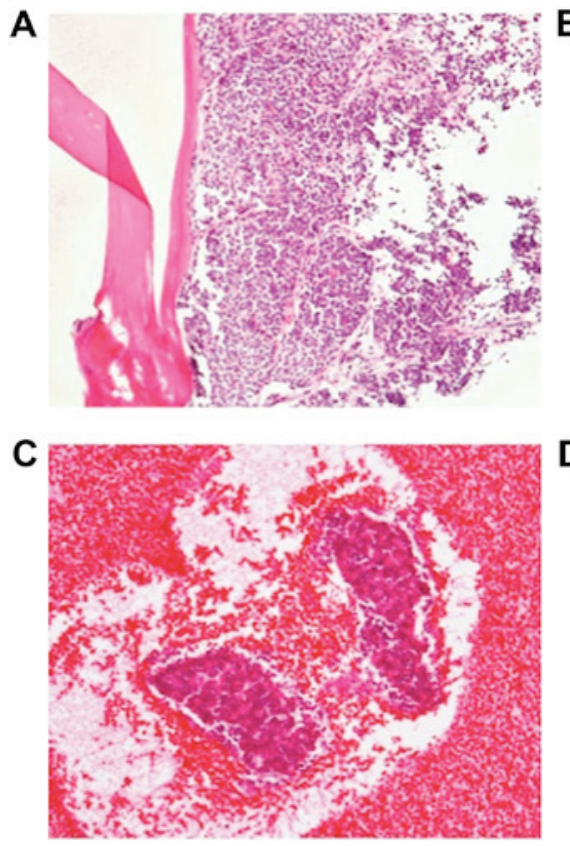

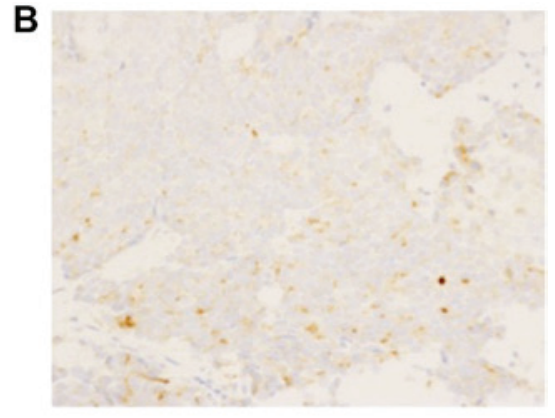

D

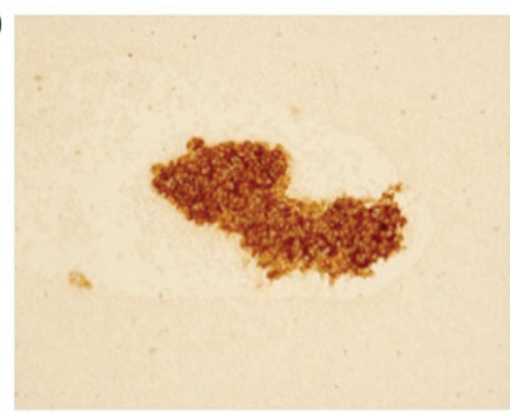

Figure 1. Histopathological findings in the two cases. (A) Hematoxylin and eosin (H\&E) staining of the bone marrow in case 1 (magnification, x10). (B) Prostate-specific antigen (PSA) immunostaining in case 1 (magnification, $\mathrm{x} 40$ ). (C) H\&E staining of the bone marrow in case 2 (magnification, $\mathrm{x} 10$ ). (D) PSA immunostaining in case 2 (magnification, $x 10$ ).

A
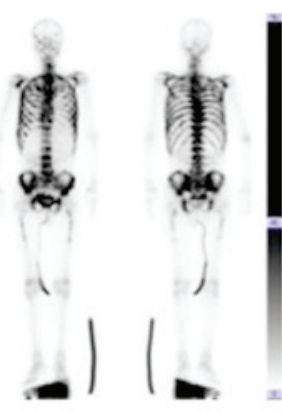

C

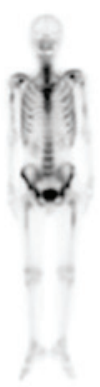

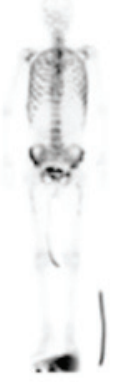
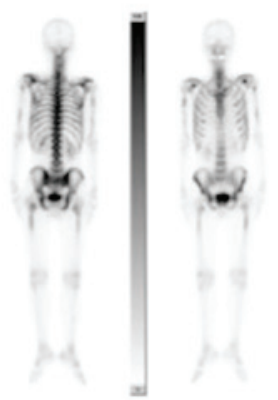

B
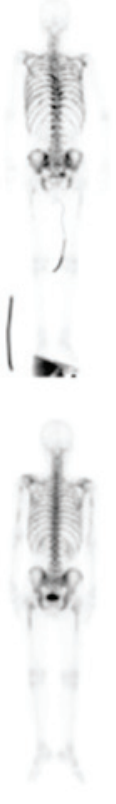

D
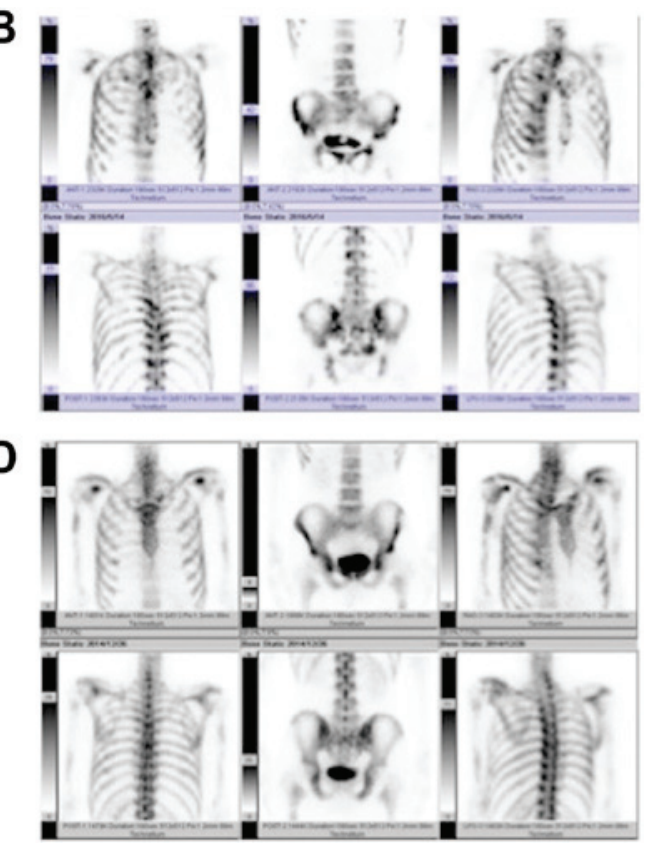

Figure 2. (A and B) Bone scintigram in case 1 showing multiple bone metastases in the spinal column, right and left ribs, shoulder blades bilaterally, the pelvic bone, and the proximal femur. (C and D) Bone scintigram in case 2 showing diffuse bone metastasis.

with enhanced fibrinolysis, the plasmin- $\alpha 2$ plasmin inhibitor complex that activates fibrinolysis is secreted at significantly higher levels compared with prothrombin activator complex, and fibrinolysis is aggravated. As a result, bleeding, rather than organ dysfunction, is the main presenting symptom. The cases presented herein also reported gingival bleeding as a main symptom, due to thrombocytopenia, and did not report other organ symptoms. Anticoagulant therapies are administered, and fresh frozen plasma and blood products are used. In addition, chemotherapy is necessary for the treatment of the underlying disease in DIC with DCBM. However, we were unable to administer the antitumor agent in sufficient therapeutic doses for patients with hematopoietic disorders. Therefore, the treatment of DIC is difficult, and it is one of the main reasons contributing to the poor prognosis of DCBM. The median survival time in 42 patients who were diagnosed with prostate cancer with DIC was 4 weeks (8).

Iguchi et al suggested a combination of bisphosphonates, including zoledronic acid, anticoagulant therapies and chemotherapies, as treatments for prostate cancer with DCBM (9). 
Bisphosphonates, including zoledronic acid, not only prevent bone destruction by inhibiting bone resorption, but also inhibit growth factor release from the bone to the bone marrow cavity and control the growth of cancer cells. In the two cases presented herein, denosumab, rather than zoledronic acid, was used for bone metastasis therapy. Denosumab is a fully human monoclonal IgG2 antibody against the receptor activator of nuclear factor- $\mathrm{KB}$ ligand (RANKL), the osteoclast differentiation factor. Denosumab is a molecular targeted drug that inhibits the differentiation and function of osteoclasts by binding to RANKL with high affinity and specificity and blocking its interactions. Denosumab was found to be superior to zoledronic acid in delaying or preventing skeletal-related events (10). In addition, denosumab has the advantage that dose adjustment depending on renal function is not required. Denosumab is associated with a higher risk of hypocalcemia and osteonecrosis of the jaw compared with zoledronic acid, and administration of an oral calcium preparation is necessary when denosumab is administered. To the best of our knowledge, the two cases presented herein are the first to be treated with denosumab for prostate cancer with DCBM. Denosumab may exert a curative effect similar to that of zoledronic acid.

Case 2 was treated with enzalutamide, a new antiandrogen agent, after the cancer had progressed to CRPC, and achieved a long-term response. Enzalutamide is distinct from the currently available antiandrogen agents in that it inhibits nuclear translocation of the androgen receptor, DNA binding, and coactivator recruitment; it also has a $\sim 5-8$-fold greater affinity for the receptor compared with other antiandrogen agents, and lacks agonist activity (11). The majority of the cases of prostate cancer with DCBM eventually become CRPC, and chemotherapy, including docetaxel, may not be able to be performed to the degree required for myelosuppression. Gauthier et al reported successful treatment of acute DIC-related CRPC with abiraterone acetate (12). Abiraterone, which inhibits the CYP17 enzyme and decreases the circulating levels of testosterone, is a newer hormonal agent similar to enzalutamide. Enzalutamide, which affects bone marrow function to a lesser extent compared with chemotherapy, is also suggested to be an effective treatment for prostate cancer with DCBM.

The two cases presented herein were diagnosed with DCBM at the first medical examination, and DIC temporarily improved with $\mathrm{CAB}$ and denosumab therapy. Case 1 became treatment-resistant, and the patient succumbed to recurrence of DIC. By contrast, in case 2, enzalutamide was effective after the patient was diagnosed with CRPC, and no recurrence of DIC has subsequently been observed. Although re-biopsy was not performed, DCBM appears to have improved. To the best of our knowledge, this report describes the first documented cases of denosumab treatment for prostate cancer with DCBM. The combination of CAB therapy and denosumab appeared to be effective in these two cases. However, the majority of the cases eventually become treatment-resistant. Patient outcomes following development of resistance to treatment may be improved by prescribing a newer hormonal agent similar to enzalutamide as soon as possible.

\section{References}

1. Hayashi H, Haruyama H, Emura Y, Kaizuka I and Ozeki T: Disseminated carcinomatpsis of thebone marrow-study of a type of metastatic cancer and relationship of microangiopathic hemolytic anemia or disseminated intravascular coagulation. Jpn J Cancer Clin 25: 329-343, 1979.

2. Jarcho S: Diffusely infiltrative carcinoma: A hitherto undescribed correlation of several varieties of tumor metastasis. Arch Pathol 22: 674-696, 1936.

3. Haratake $\mathbf{J}$ and Horie A: Clinicopathological examination of 12 autopsy cases of carcinomatosis of the bone marrow. Gan No Rinsho 31: 168-178, 1985 (In Japanese).

4. Smith JA, Jr., Soloway MS and Young MJ: Complications of advanced prostate cancer. Urology 54 (6A Suppl): S8-S14, 1999.

5. Chernow B and Wallner SF: Variables predictive of bone marrow metastasis. Cancer 42: 2373-2378, 1978.

6. Japanese Society of Medical Oncology: Practical guideline of bone metastasis. Nankodo Co., Ltd., Tokyo, 2015 (In Japanese).

7. Sallah S, Wan JY, Nguyen NP, Hanrahan LR and Sigounas G: Disseminated intravascular coagulation in solid tumors: Clinical and pathologic study. Thromb Haemost 86: 828-833, 2001.

8. Hyman DM, Soff GA and Kampel LJ: Disseminated intravascular coagulation with excessive fibrinolysis in prostate cancer: A case series and review of the literature. Oncology 81: 119-125, 2011.

9. Iguchi $\mathrm{H}$ and Matsuhisa T: Characteristic features of bone disease in disseminated carcinomatosis of bone marrow. Nihon Rinsho (65 Suppl 9): S550-S554, 2007 (In Japanese).

10. Fizazi K, Carducci M, Smith M, Damião R, Brown J, Karsh L, Milecki P, Shore N, Rader M, Wang H, et al: Denosumab versus zoledronic acid for treatment of bone metastases in men with castration-resistant prostate cancer: A randomised, double-blind study. Lancet 377: 813-822, 2011.

11. Tran C, Ouk S, Clegg NJ, Chen Y, Watson PA, Arora V, Wongvipat J, Smith-Jones PM, Yoo D, Kwon A, et al: Development of a second-generation antiandrogen for treatment of advanced prostate cancer. Science 324: 787-790, 2009.

12. Gauthier H, Serrate C, Pouessel D, le Maignan C, Teixeira L and Culine S: Very fast recovery of acute disseminated intravascular coagulation with abiraterone acetate in a patient with bone metastases from castrate-resistant prostate cancer. Case Rep Oncol 7: 625-627, 2014. 